\title{
A MIXED NORM VARIANT OF WOLFF'S INEQUALITY FOR PARABOLOIDS
}

\author{
GUSTAVO GARRIGÓS AND ANDREAS SEEGER
}

\begin{abstract}
We adapt the proof for $\ell^{p}\left(L^{p}\right)$ Wolff inequalities in the case of plate decompositions of paraboloids, to obtain stronger $\ell^{2}\left(L^{p}\right)$ versions. These are motivated by the study of Bergman projections for tube domains.
\end{abstract}

\section{Introduction And STATEMENT of RESUlts}

For small $\delta>0$, let $\Sigma^{\delta}$ denote a truncated $\delta$-neighborhood of the paraboloid in $\mathbb{R}^{d}$,

$$
\Sigma^{\delta} \equiv\left\{\xi=\left(\xi^{\prime}, \xi_{d}\right) \in \mathbb{R}^{d}:\left.\left|\xi_{d}-\right| \xi^{\prime}\right|^{2} / 2|\leq \delta,| \xi^{\prime} \mid \leq 1\right\} .
$$

Consider the usual covering of $\Sigma^{\delta}$ by $C\left(\delta^{1 / 2} \times{ }^{(d-1)} \times \delta^{1 / 2} \times \delta\right)$-plates, $\Pi_{k}^{(\delta)}$, subordinated to a $\sqrt{\delta}$-separated sequence $\left\{y_{k}\right\} \subset \mathbb{R}^{d-1}$; namely $\operatorname{dist}\left(y_{k}, y_{k^{\prime}}\right) \geq \sqrt{\delta}$ if $k \neq k^{\prime}$, and

$$
\Pi_{k}^{(\delta)}=\left\{\left(\xi^{\prime}, \xi_{d}\right) \in \Sigma_{\delta}:\left|\xi^{\prime}-y_{k}\right| \leq C^{\prime} \sqrt{\delta}\right\} .
$$

Typically $y_{k}=k \sqrt{\delta}$ for $k \in \mathbb{Z}^{d-1}$ with $|k| \leq \delta^{-1 / 2}$.

In this paper we are interested in the validity of the inequality

$$
\begin{aligned}
& \left\|\sum_{k} f_{k}\right\|_{p} \leq C_{\varepsilon} \delta^{-\beta(p)-\varepsilon}\left(\sum_{k}\left\|f_{k}\right\|_{p}^{2}\right)^{1 / 2}, \quad \text { for all }\left\{f_{k}\right\} \text { with supp } \widehat{f}_{k} \subset \Pi_{k}^{(\delta)}, \\
& \text { where } \beta(p)=\frac{d-1}{4}-\frac{d+1}{2 p} .
\end{aligned}
$$

Theorem 1.1. Let $d \geq 2$. Then, for all $\varepsilon>0$ the mixed norm inequality (1.3) holds when $p \geq p_{d, *}=2+\frac{8}{d-1}-\frac{4}{d(d-1)}$.

The power $-\beta(p)-\varepsilon$ is best possible (except perhaps for $\varepsilon>0$ ) but the range is not, indeed (1.3) is conjectured to hold for all $p \geq 2+\frac{4}{d-1}$. The problem is motivated by questions on the Bergman projection for tube domains over light cones [1] where a similar inequality for plate decomposition of neighborhoods of cones plays a crucial role. This harder inequality is considered in [4].

Inequality (1.3) is a mixed norm variant of a Wolff inequality for paraboloids which itself can be considered as a model problem simplifying the corresponding harder problem for

Date: August 14, 2008.

First author partially supported by grant MTM2007-60952 and Programa Ramón y Cajal MCyT (Spain). Second author partially supported by NSF grant DMS 0652890. 
decompositions of cone multipliers in $\mathbb{R}^{d+1}$ (see [13], [7], [5], [4]). Let $\alpha(p):=d\left(\frac{1}{2}-\frac{1}{p}\right)-\frac{1}{2}$, the standard Bochner-Riesz critical index in $d$ dimensions. Then Wolff's inequality for paraboloids asserts that for all $\varepsilon>0$

$$
\left\|\sum_{k} f_{k}\right\|_{p} \leq C_{\varepsilon} \delta^{-\alpha(p)-\varepsilon}\left(\sum_{k}\left\|f_{k}\right\|_{p}^{p}\right)^{1 / p}, \quad \text { for all }\left\{f_{k}\right\} \text { with supp } \widehat{f_{k}} \subset \Pi_{k}^{(\delta)} \text {. }
$$

As before, the power $\alpha(p)$ is optimal for each $p$ (except for $\varepsilon>0$ ), and the inequality is conjectured to hold for all $p>2+\frac{4}{d-1}$. By an interpolation argument the inequality (1.4) for some $\widetilde{p}$ implies the mixed norm variant (1.3) in the smaller range $p>2(\widetilde{p}-1)$ only. On the other hand, inequality (1.4) for fixed $p$ is implied by (1.4) for the same $p$, by Hölder's inequality, since $\alpha(p)-\beta(p)=\frac{d-1}{2}\left(\frac{1}{2}-\frac{1}{p}\right)$. Theorem 1.1 states that the stronger mixed norm inequality holds in the same range as the currently known range for the Wolff inequality (1.4) ( $c f$. [5]), that is for $p \geq 2+\frac{8}{d-1}-\frac{4}{d(d-1)}$. We also remark that the resolution of the problem for the paraboloid is necessary for the corresponding problems for cones in $\mathbb{R}^{d+1}$.

By a randomization argument it is easy to see that the conjectured range $p \geq 2+4 /(d-1)$ is sharp for (1.4) (and a fortiori for (1.3)). Let $\left\{r_{k}\right\}$ be the sequence of Rademacherfunctions on $[0,1]$ and define $h_{k}$ by $\widehat{h}_{k}(\xi)=\varphi\left(\delta^{-1}\left(\xi-\omega_{k}\right)\right)$ for a $C^{\infty}$ function $\varphi$ supported in $\{|\xi| \leq 1 / 10\}$, and where $\omega_{k}=\left(y_{k},\left|y_{k}\right|^{2} / 2\right)$. Let $h_{k, t}(x)=r_{k}(t) h_{k}(x)$ for $t \in[0,1]$. Then the validity of Wolff's inequality implies that

$$
\left(\int_{0}^{1}\left\|\sum h_{k, t}\right\|_{p}^{p} d t\right)^{1 / p} \lesssim \delta^{-\alpha(p)+\epsilon}\left(\sum_{k}\left\|h_{k}\right\|_{p}^{p}\right)^{1 / p}
$$

and by Fubini's theorem and the familiar inequality for Rademacher functions ([10])

$$
\left\|\left(\sum_{k}\left|h_{k}\right|^{2}\right)^{1 / 2}\right\|_{p} \lesssim \delta^{-\alpha(p)-\epsilon}\left(\sum_{k}\left\|h_{k}\right\|_{p}^{p}\right)^{1 / p}
$$

This leads to $\delta^{-(d-1) / 4} \lesssim \delta^{-\alpha(p)-\varepsilon} \delta^{-(d-1) /(2 p)}$ and consequently to the restriction $p \geq \frac{2(d+1)}{d-1}$.

Returning to (1.3), there is a square-function variant with a larger exponent,

$$
\left\|\sum_{k} h_{k}\right\|_{L^{q}\left(\mathbb{R}^{d}\right)} \leq C_{q, \varepsilon} \delta^{-(\varepsilon+\alpha(q) / 2)}\left\|\left(\sum_{k}\left|h_{k}\right|^{2}\right)^{1 / 2}\right\|_{L^{q}\left(\mathbb{R}^{d}\right)}, \quad \forall\left\{h_{k}\right\} \quad: \operatorname{supp} \widehat{h_{k}} \subset \Pi_{k}^{(\delta)},
$$

which is known to hold in some range of $q \leq 2(d+1) /(d-1)$. For $q \geq 2(d+1) /(d-1)$ inequality (1.5) with $\varepsilon=0$ is a consequence of the Stein-Tomas adjoint restriction theorem as was shown by Bourgain [2]. In two dimensions the inequality in the optimal range $q \geq 4$, again with $\varepsilon=0$, is due to Fefferman [3], and the proof of the crucial $L^{4}$ bound is based on the observation that in two dimensions the algebraic sums of plates $\Pi_{k}^{(\delta)}+\Pi_{k^{\prime}}^{(\delta)}$ are essentially disjoint as $\left(k, k^{\prime}\right)$ run over integers with $|k|,\left|k^{\prime}\right| \lesssim \delta^{-1 / 2}$. In dimensions $d \geq 3$ it is conjectured, but not known, that (1.5) holds on $L^{q_{0}}\left(\mathbb{R}^{d}\right)$ with $q_{0}=2 d /(d-1)$. Partial 
results in higher dimensions follow from the bilinear adjoint restriction theorem of Tao [11] using arguments in [8], [5]; indeed (1.5) is known to hold for $q>2+4 / d$.

By Minkowski's inequality the square function bound (1.5) also implies the weaker (and possibly non optimal)

$$
\left\|\sum_{k} h_{k}\right\|_{L^{q}\left(\mathbb{R}^{d}\right)} \leq C_{\varepsilon} \delta^{-(\alpha(q)+\varepsilon) / 2}\left(\sum_{k}\left\|h_{k}\right\|_{L^{q}\left(\mathbb{R}^{d}\right)}^{2}\right)^{1 / 2}, \quad \operatorname{supp} \widehat{h_{k}} \subset \Pi_{k}^{(\delta)} .
$$

We use inequality (1.6) as a hypothesis:

Definition 1.2. Suppose $2 d /(d-1)<q \leq 2(d+1) /(d-1)$. We say that Hypothesis $S(2, q)$ holds if (1.6) holds for all $\varepsilon>0$.

Under this hypothesis we show

Theorem 1.3. Suppose $d \geq 2$ and $2 d /(d-1)<q \leq 2(d+1) /(d-1)$ and Hypothesis $S(2, q)$ holds. Then the inequality (1.3) holds for all $p \geq p_{d}:=q+4 /(d-1)$.

Theorem 1.1 follows from Theorem 1.3 since, as pointed out above, $S(2, q)$ holds for $q>2+4 / d$. If one could prove the above square function estimate in the optimal range $q>2 d /(d-1)$ (and therefore $S(2, q)$ in the same range) then the range of (1.3) would improve to $p \geq 2+6 /(d-1)$.

A reformulation. Let $\zeta$ be a function in $C_{c}^{\infty}\left(\mathbb{R}^{d-1}\right)$ which is identically 1 in the cube $\left\{\xi^{\prime}:\left|\xi_{i}\right| \leq 1, i=1, \ldots, d-1\right\}$, and let $\zeta_{0}$ be a Schwartz function on $\mathbb{R}$ with compact support in $(-2,2)$ so that $\zeta_{0}(\tau)=1$ for $|\tau| \leq 1$. For $k \in \mathbb{Z}^{d-1},|k| \lesssim \delta^{-1 / 2}$ define operators $P_{k}=P_{k}^{(\delta)}$ by

$$
\widehat{P_{k} f}(\xi)=\zeta\left(\delta^{-1 / 2} \xi^{\prime}-k\right) \zeta_{0}\left(\delta^{-1}\left(\xi_{d}-\left|\xi^{\prime}\right|^{2} / 2\right)\right) \widehat{f}(\xi) .
$$

Note that with the choice of $y_{k}=\delta^{1 / 2} k$ the supports of the functions $\widehat{P_{k} f}$ are essentially the plates $\Pi_{k}^{(\delta)}$ (actually slightly expanded plates).

The operators $P_{k}$ are uniformly bounded on all $L^{p}$ (as long as $|k| \lesssim \delta^{-1 / 2}$ ) and (1.3) is equivalent with the statement that for all families of $L^{p}$ functions $\left\{h_{k}\right\}$

$$
\left\|\sum_{|k| \lesssim \delta^{-1 / 2}} P_{k} h_{k}\right\|_{p} \leq C_{\varepsilon} \delta^{-\beta(p)-\varepsilon}\left(\sum_{k}\left\|h_{k}\right\|_{p}^{2}\right)^{1 / 2} .
$$

For functions with Fourier transform supported in $\Sigma^{\delta}$ we may define a norm

$$
\|f\|_{p, 2 ; \delta}=\left(\sum_{k}\left\|P_{k} f\right\|_{p}^{2}\right)^{1 / 2}
$$

Note that if $f=\sum f_{k}$ with $\operatorname{supp} \widehat{f}_{k} \subset \Pi_{k}^{\delta}$ we have

$$
\|f\|_{p, 2 ; \delta} \approx\left(\sum_{k}\left\|f_{k}\right\|_{p}^{2}\right)^{1 / 2} .
$$


More general surfaces. Theorem 1.1 may be extended to convex surfaces with nonvanishing Gaussian curvature, using arguments in $\S 2$ of [9]. Namely, one notes that on sets of diameter $\gamma^{1 / 3} \ll 1$ the surface can be approximated by paraboloids with accuracy $O(\gamma)$ and uses the scaled estimate in $\S 5$ below, together with an induction on scales argument. One could also modify the proof for paraboloids using arguments in [6] (which apply to more general situations).

Acknowledgements. The main ideas can be traced back to the pioneering work by Wolff [13], see also the subsequent articles [7], [9], [6], [5] and [4]. An earlier version of this paper was originally written as class notes intended to give an expository account of some of the material in [13] and [7]. For self-containedness and in order to retain the expository nature of the notes we have included in $\S 4$ material from Laba-Wolff [7] which could have been quoted. We are indebted to Wilhelm Schlag for comments and for collaboration on [4] and to Detlef Müller for useful remarks on an earlier version of this paper.

\section{Notation AND BASiC DEFinitions}

We note that because of the appearance of $\varepsilon$ in (1.3) we may assume that $p>p_{d}=$ $q+4 /(d-1)$ since we can then interpolate with a trivial $\ell^{2}\left(L^{2}\right)$ inequality to get the result for $p=p_{d}$.

Throughout we fix $p>p_{d}$. We also fix a positive but very small $\varepsilon_{0}$, which may depend on $p$ and $q$ and will be determined later. We remark that for the proof of Theorem 1.3 in the range $p>p_{d}=q+4 /(d-1)$ the choice

$$
\varepsilon_{0}=10^{-3} d^{-1}(d-1-4 /(p-q))
$$

is admissible. Statements involving the parameter $\delta$ are assumed to hold for all $\delta \in\left(0, \delta_{0}\right]$, for some fixed $\delta_{0} \ll 1$. For each such $\delta$ we set

$$
N=1 / \delta \quad \text { and } t=\delta^{\epsilon_{0}}=N^{-\epsilon_{0}} .
$$

The constants $C, c_{0}, c_{1}, \ldots$ appearing below may depend on $p, d, \varepsilon_{0}, \delta_{0}$ and also on other constants appearing below, but will be independent of $\delta, f_{k},\left\{y_{k}\right\}$, and parameters such as $\lambda$ or $\varepsilon$. Otherwise we will indicate it by $C_{\varepsilon}$, etc... By $A \lesssim B$ we will mean $A \leq C B$ for some $C$ as above, and by $A \lesssim B$ we mean $A \leq C(\log N)^{C} B$, for some $C>0$. We shall write either $\operatorname{card}(\mathcal{P})$ or $\# \mathcal{P}$ for the cardinality of a finite set $\mathcal{P}$, and meas $(A)$ or $|A|$ for the Lebesgue measure of a set in $\mathbb{R}^{d}$.

Plates and plate families. A rectangular box in $\mathbb{R}^{d}$ of size $\sqrt{N} \times{ }_{\ldots}^{(d-1)} \times \sqrt{N} \times N$. will be referred to as an $N$-plate. We typically denote plates in $x$-space by $\pi$ and plate families by $\mathcal{P}$. We shall always assume that $N$-plates are essentially dual to some $\Pi_{k}^{(\delta)}$. In this case we 
use the notation

$$
\pi \| k
$$

to indicate that $\pi$ is an $N$-plate, whose long side is parallel to $\mathfrak{n}_{k}=\left(y_{k},-1\right)=(k \sqrt{\delta},-1)$. Observe that, for different $k$ 's, plate directions are $\sqrt{\delta}$-separated, since so are the directions of $\left\{\mathfrak{n}_{k}\right\}$. The integer vectors $k$ will be taken in

$$
\mathcal{Z}(\sqrt{N})=\left\{k \in \mathbb{Z}^{d-1}:\left|k_{i}\right| \leq \sqrt{N}, i=1, \ldots, d-1\right\} .
$$

We shall also assume that families $\mathcal{P}$ consist only of separated plates, meaning that for each $\pi \in \mathcal{P}$ at most $C_{1}$ plates from $\mathcal{P}$ can be contained in a fixed dilate $C_{2} \pi$, where $C_{1}$ and $C_{2}$ are fixed universal constants. This means that for fixed $k$, plates $\pi \| k$ are essentially disjoint.

We recall that the cardinality of $\mathcal{Z}(\sqrt{N})$, and thus the number of essentially different directions that plates can achieve at scale $N$, is approximately $N^{\frac{d-1}{2}}$. Finally, a $\sigma$-cube $\Delta$ is a cube of sidelength $\sigma$ centered at some point of the grid $\sigma \mathbb{Z}^{d}$.

Localizing weight functions. Given a fixed large $M$ we let

$$
w(x)=\left(1+|x|^{2}\right)^{-M / 2},
$$

and given a rectangle $R$ we denote $w_{R}=w \circ a_{R}^{-1}$, where $a_{R}$ is an affine map taking the unit cube centered at 0 to the rectangle $R$. Thus $w_{R}$ is roughly the characteristic function of $R$ with "Schwartz tails" (with an abuse of language as for fixed $M$ the function $w$ is not a Schwartz-function).

We shall also use a fixed Schwartz function $\psi$, strictly positive in $B_{2}(0)$, with Fourier transform supported in $B_{\frac{1}{100}}(0)$, and so that $\sum_{n \in \mathbb{Z}^{d}} \psi^{2}(\cdot+n)=1$. Again we set

$$
\psi_{R}=\psi \circ a_{R}^{-1} .
$$

In particular, if $\{\Delta\}$ is a tiling of $\mathbb{R}^{d}$ by $\sigma$-cubes with centers $c_{\Delta}$ in $\sigma \mathbb{Z}^{d}$, then $\sum_{\Delta} \psi_{\Delta}^{2}=1$, where $\psi_{\Delta}(x)=\psi\left(\left(x-c_{\Delta}\right) / \sigma\right)$.

Elementary properties of $\|\cdot\|_{p, 2 ; \delta}$.

Lemma 2.1. Let $2 \leq p \leq \infty$ and $\widehat{f}$ be supported in $\Sigma^{\delta}$. Then

$$
\begin{aligned}
\|f\|_{\infty, 2 ; \delta} & \lesssim N^{-(d+1) / 2 p}\|f\|_{p, 2 ; \delta} \\
\|f\|_{\infty} & \lesssim N^{\beta(p)}\|f\|_{p, 2 ; \delta} \\
\|f\|_{p, 2 ; \delta} & \lesssim\|f\|_{2}^{2 / p}\|f\|_{\infty, 2 ; \delta}^{1-2 / p}
\end{aligned}
$$

Proof. If $\widehat{g}$ is supported in $\Pi_{k}^{(\delta)}$ then by Young's inequality $\|g\|_{\infty} \lesssim N^{-(d+1) / 2 p}\|g\|_{p}$; this yields (2.5). If $f=\sum f_{k}$ with $\widehat{f}_{k}$ supported in $\Pi_{k}^{(\delta)}$ then

$$
\|f\|_{\infty} \lesssim \sum_{k}\left\|f_{k}\right\|_{\infty} \lesssim N^{\frac{d-1}{4}}\left(\sum_{k}\left\|f_{k}\right\|_{\infty}^{2}\right)^{1 / 2} \lesssim N^{\frac{d-1}{4}-\frac{d+1}{2 p}}\left(\sum_{k}\left\|f_{k}\right\|_{p}^{2}\right)^{1 / 2}
$$


which is (2.6). Inequality (2.7) follows from a corresponding interpolation inequality for the projection operators $P_{k}$, namely for $\vartheta=1-2 / p$,

$$
\left(\sum_{k}\left\|P_{k} h_{k}\right\|_{p}^{2}\right)^{1 / 2} \lesssim\left(\sum_{k}\left\|h_{k}\right\|_{2}^{2}\right)^{(1-\vartheta) / 2}\left(\sum_{k}\left\|h_{k}\right\|_{\infty}^{2}\right)^{\vartheta / 2} .
$$

This follows by convexity from the obvious cases $p=2$ and $p=\infty$.

We also need the following localization estimate.

Lemma 2.2. Let $\widehat{f}$ be supported in $\Sigma^{\delta}$. Let $\mathcal{Q}=\{Q\}$ be a grid of $N$-cubes and let $\psi_{Q}$ be as in (2.4) (so that $\widehat{\psi}_{Q}$ is supported in $|\xi| \leq(100 N)^{-1}$ ). Then

$$
\left(\sum_{Q}\left\|\psi_{Q} f\right\|_{p, 2 ; 2 \delta}^{p}\right)^{1 / p} \lesssim\|f\|_{p, 2 ; \delta}
$$

Proof. Note that $\widehat{\psi_{Q}} * \widehat{f}$ is supported in $\Sigma^{2 \delta}$. The case $p=\infty$ is immediate and the case $p=2$ follows by orthogonality. One uses the projection operators $P_{k}$ to set up an interpolation argument showing the inequality for $2<p<\infty$.

Packets.

Definition 2.3. (i) $f$ is called an $N$-packet associated with $\Pi_{k}^{(\delta)}$ if it can be written as $f=\sum_{\pi \in \mathcal{P}} f_{\pi}$ for some family $\mathcal{P}=\mathcal{P}_{k}(f)$ of separated $N$-plates with $\pi \| k$, in such a way that every $f_{\pi}, \pi \in \mathcal{P}_{k}$, satisfies

$$
\left|f_{\pi}\right| \leq c_{1} w_{\pi} \quad \text { and } \quad \operatorname{supp} \widehat{f_{\pi}} \subset c_{2} \Pi_{k}^{(\delta)} .
$$

(ii) Let $R$ be a cube of diameter $\geq N$ and let $E \subset \mathcal{Z}(\sqrt{N})$. An $(N, R, E)$-packet $f$ is a function that can be written as

$$
f=\frac{1}{\sqrt{\# E}} \sum_{k \in E} \sum_{\pi \in \mathcal{P}_{k}} f_{\pi}
$$

where $\mathcal{P}_{k}$ consists of plates $\pi \| k$ which have nonempty intersection with $R$, and $f_{\pi}$ are functions so that (2.9) holds for all $\pi \in \mathcal{P}_{k}$ and all $k \in E$. We denote by $\mathcal{P}(f)=\cup_{k \in E} \mathcal{P}_{k}$ the plate family of $f$.

(iii) For $f$ as in (ii), we say that $g$ is a subpacket of $f$ if $g=\frac{1}{\sqrt{\# E}} \sum_{k \in E} \sum_{\pi \in \mathcal{P}_{k}^{\prime}} f_{\pi}$, with $\mathcal{P}_{k}^{\prime} \subset \mathcal{P}_{k}$.

(iv) An $(N, R, E)$-packet $f$ as in $(2.10)$ is called stable if it satisfies

$$
\frac{1}{2} \# \mathcal{P}_{k} \leq \# \mathcal{P}_{k^{\prime}} \leq 2 \# \mathcal{P}_{k} \quad \text { whenever } k, k^{\prime} \in E .
$$

Elementary properties of packets are listed in 
Lemma 2.4. Let $f$ be an $(N, Q, E)$-packet. Then

$$
\begin{gathered}
\|f\|_{\infty, 2 ; \delta} \lesssim 1 \\
\|f\|_{\infty} \lesssim \sqrt{\# E} \lesssim N^{(d-1) / 4},
\end{gathered}
$$

and, for $2 \leq p<\infty$

$$
\|f\|_{p, 2 ; \delta}^{p} \leq C_{p} \frac{N^{(d+1) / 2} \# \mathcal{P}(f)}{\# E}
$$

Proof. The bounds (2.12) and (2.13) are immediate. We can use the interpolation inequality $\|G\|_{\ell^{2}\left(L^{p}\right)} \leq\|G\|_{\ell^{2}\left(L^{2}\right)}^{2 / p}\|G\|_{\ell^{2}\left(L^{\infty}\right)}^{1-2 / p}$ to see that (2.14) follows from the (2.12) and the case $p=2$ of (2.14). Observe that $\|f\|_{2,2 ; \delta}^{2}$ is dominated by

$$
\sum_{k \in E}\left\|\sum_{\pi \in \mathcal{P}_{k}} \frac{f_{\pi}}{\sqrt{\# E}}\right\|_{2}^{2} \lesssim \frac{1}{\# E} \sum_{k \in E}\left\|\sum_{\pi \in \mathcal{P}_{k}} w_{\pi}\right\|_{2}^{2} \lesssim \frac{1}{\# E} \sum_{k \in E} N^{\frac{d+1}{2}} \# \mathcal{P}_{k}
$$

and the last expression is equal to $N^{(d+1) / 2} \# \mathcal{P}(f) / \# E$.

Another preparatory result concerns decompositions of functions with Fourier support in $\Sigma^{\delta}$ into (stable) $N$-packets. The stability property (2.11) gives estimate $(2.18)$ in the following lemma (a sort of converse to (2.14)), which will be crucial in the induction on scales argument, $c f$. Lemma 6.1 below.

Lemma 2.5. Let $\widehat{f}$ be supported in $\Sigma^{\delta}$ and assume that

$$
\|f\|_{\infty, 2 ; \delta} \leq A .
$$

Let $Q$ be an $N$-cube, let $0<\varepsilon \leq 1$, and let $R$ be the cube of sidelength $N^{1+\varepsilon}$ with the same center as $Q$. Then on $Q$ we may decompose

$$
f(x)=\sum_{A N^{-10 d} \leq 2^{j} \leq C_{\varepsilon} A} 2^{j} \sum_{\ell=1}^{n_{j}} f_{j, \ell}(x)+g(x), \quad x \in Q,
$$

for some integers $n_{j} \leq C_{\varepsilon}(\log N)^{2}$, and where

(i) the function $g$ satisfies

$$
\sup _{x \in Q}|g(x)| \leq C_{\varepsilon} N^{-8 d} A ;
$$

(ii) for each $j, \ell$ the function $f_{j, \ell}$ is a stable $\left(N, R, E_{j, \ell}\right)$-packet, for some subset $E_{j, \ell}$ of $\mathcal{Z}\left(N^{1 / 2}\right)$, and with associated plate family $\mathcal{P}^{j, \ell}$ containing only plates $\pi$ with $\operatorname{dist}(Q, \pi) \leq$ $N^{1+\varepsilon}$ 
(iii) for every $2 \leq p \leq \infty$ and every $j, \ell$ it holds

$$
2^{j}\left(N^{\frac{d+1}{2}} \# \mathcal{P}^{j, \ell}\right)^{1 / p} \lesssim\|f\|_{p, 2 ; \delta}\left(\# E_{j, \ell}\right)^{1 / p} .
$$

Proof. We decompose $f=\sum_{k} f_{k}$ where $\widehat{f}_{k}$ is supported in $\Pi_{k}^{(\delta)}$. By a pidgeonhole argument we may immediately reduce to the case where the $k$ are strongly separated, in the sense that, if $k$ and $k^{\prime}$ occur in the sum and are different then $\left|k-k^{\prime}\right| \geq 10 d$. Note that then

$$
\|f\|_{p, 2 ; \delta} \approx\left(\sum_{k}\left\|f_{k}\right\|_{p}^{2}\right)^{1 / 2} .
$$

Next, we fix $k$, and further decompose $f_{k}$ as

$$
f_{k}=\sum_{\pi \| k} f_{k} \psi_{\pi}^{2}
$$

We let $\mathcal{P}_{k} \equiv \mathcal{P}_{k, R}(f)$ be the family of all $\pi$ with $\pi \| k$ and which intersect $R$. Notice that there are at most $O\left(N^{d(1+\varepsilon)}\right)$ in $\cup_{k} \mathcal{P}_{k, R}(f)$.

We first discard the terms involving plates that do not intersect $R$. Let

$$
g_{\text {compl }}(x)=\sum_{k} \sum_{\substack{\pi \| k \\ \pi \cap R=\emptyset}} f_{k} \psi_{\pi}^{2} .
$$

Using the rapid decay of the functions $w_{\pi}$ away from $\pi$ we get

$$
\left\|g_{\text {compl }}\right\|_{L^{\infty}(Q)} \lesssim N^{(d-1) / 4}\|f\|_{\infty, 2 ; \delta} \sup _{x \in Q}\left(\sum_{k} \sum_{\substack{\pi \| k \\ \pi \cap R=\emptyset}}\left|w_{\pi}(x)\right|^{2}\right)^{1 / 2} \leq C_{\varepsilon} A N^{\frac{d-1}{2}-M \varepsilon}
$$

and here $M$ (in the definition of (2.3)) may be chosen so large that $M \varepsilon>10 d$.

Secondly we discard terms for which $\pi$ intersects $R$ but $\left\|f_{k} \psi_{\pi}\right\|_{\infty}$ is very small. Define

$$
g_{\text {small }}(x)=\sum_{k} \sum_{\substack{\pi \in \mathcal{P}_{k, R} \\\left\|f_{k} \psi_{\pi}\right\|_{\infty} \leq A N^{-10 d}}} f_{k} \psi_{\pi}^{2}
$$

As the cardinality of all plates intersecting $R$ is $O\left(N^{d(1+\varepsilon)}\right)$ we trivially get

$$
\left\|g_{\text {small }}\right\|_{L^{\infty}(Q)} \lesssim N^{2 d} A N^{-10 d}
$$

and if we set $g=g_{\text {small }}+g_{\text {compl }}$ the bound (2.17) follows.

It remains to decompose the function

$$
f-g_{\text {small }}-g_{\text {compl }}=\sum_{k} \sum_{\substack{\pi \in \mathcal{P}_{k}, R \\\left\|f_{k} \psi_{\pi}\right\|_{\infty}>A N^{-10 d}}} f_{k} \psi_{\pi}^{2} .
$$

Note that $\|f\|_{\infty} \lesssim A N^{(d-1) / 4}$ (by $(2.6)$ for $p=\infty$ ) so that there are only $O(\log N)$ relevant dyadic scales for the possible size of $\left\|f \psi_{\pi}\right\|_{\infty}$. 
For each $k$ define

$$
\mathcal{P}_{k, R}^{m}=\left\{\pi \in \mathcal{P}_{k, R}: 2^{m}<\left\|f_{k} \psi_{\pi}\right\|_{\infty} \leq 2^{m+1}\right\}
$$

Next, for $i=0,1,2, \ldots$, define

$$
E(i, m)=\left\{k \in \mathcal{Z}(\sqrt{N}): 2^{i} \leq \# \mathcal{P}_{k, R}^{m}<2^{i+1}\right\} ;
$$

clearly these sets are disjoint subsets of $\mathcal{Z}(\sqrt{N})$. Set

$$
F^{i, m}=\sum_{k \in E(i, m)} \sum_{\pi \in \mathcal{P}_{k, R}^{m}} f_{k} \psi_{\pi}^{2}
$$

Notice that by definition the cardinalities of $\mathcal{P}_{k, R}^{m}$ are comparable for $k \in E(i, m)$. If we divide $F^{i, m}$ by $C 2^{m} \sqrt{\# E(i, m)}$, for suitably large $C$, then the new function will be a stable $(N, R, E(i, m))$ packet.

Recall $A N^{-10 d} \leq C 2^{m} \sqrt{\# E(i, m)} \lesssim A N^{d}$. Now for each $j$ with $A N^{-10 d} \leq C 2^{j} \lesssim A N^{d}$ there are $n_{j}=O\left((\log N)^{2}\right)$ pairs $(i, m)$ with

$$
2^{j-1}<C 2^{m} \sqrt{\# E(i, m)} \leq 2^{j}
$$

for these $(i, m)$ the functions $2^{-j} F^{i, m}$ are also stable $(N, R, E(i, m))$-packets.

We relabel these $n_{j}$ functions as $f_{j, \ell}, \ell=1, \ldots, n_{j}$, the associated plate families as $\mathcal{P}^{j, \ell}$ and the associated sets $E(i, m)$ of directions as $E_{j, \ell}$ and then obtain the decomposition $f=\sum_{2^{j} \geq A N^{-10 d}} \sum_{\ell=1}^{n_{j}} 2^{j} f_{j, \ell}+g$, for $x \in Q$.

If $\mathcal{P}_{k}^{j, \ell}=\left\{\pi \in \mathcal{P}^{j, \ell}: \pi \| k\right\}$ then by construction

$$
\# \mathcal{P}^{j, \ell} \approx\left(\# E_{j, \ell}\right)\left(\# \mathcal{P}_{k}^{j, \ell}\right)
$$

We use this to verify $(2.18)$. Fix $(j, \ell)$, and with the above notation assume $E_{j, \ell}=E(i, m)$. Then we observe

$$
\begin{aligned}
& \|f\|_{p, 2, \delta} \gtrsim\left(\sum_{k}\left\|f_{k}\right\|_{p}^{2}\right)^{1 / 2} \gtrsim\left(\sum_{k}\left(\sum_{\pi \| k}\left\|f_{k} \psi_{\pi}\right\|_{p}^{p}\right)^{2 / p}\right)^{1 / 2} \\
& \quad \geq\left(\sum_{k \in E(i, m)}\left(\sum_{\pi \in \mathcal{P}_{k, R}^{m}}\left\|f_{k} \psi_{\pi}\right\|_{p}^{p}\right)^{2 / p}\right)^{1 / 2} \gtrsim\left(\sum_{k \in E(i, m)}\left(\sum_{\pi \in \mathcal{P}_{k, R}^{m}}\left\|f_{k} \psi_{\pi}\right\|_{\infty}^{p} N^{(d+1) / 2}\right)^{2 / p}\right)^{1 / 2} \\
& \quad \gtrsim\left(\sum_{k \in E(i, m)}\left(2^{m p} \# \mathcal{P}_{k, R}^{m} N^{(d+1) / 2}\right)^{2 / p}\right)^{1 / 2} \geq\left(\sum_{k \in E_{j, \ell}}\left(2^{m p} \frac{\# \mathcal{P}^{j, \ell}}{\# E_{j, \ell}} N^{(d+1) / 2}\right)^{2 / p}\right)^{1 / 2} \\
& \quad \gtrsim 2^{m}\left(\# E_{j, \ell}\right)^{1 / 2-1 / p}\left(\# \mathcal{P}^{j, \ell}\right)^{1 / p} N^{(d+1) / 2 p},
\end{aligned}
$$

and from (2.25) we obtain (2.18). We note that (2.18) for $p=\infty$ also shows that the sum in $j$ in (2.16) is restricted to the range $2^{j} \leq C_{\varepsilon} A$. 


\section{Equivalent Formulations of the PROBlem}

We continue to assume that always $p>\frac{2(d+1)}{d-1}$ and that $S(2, q)$ holds for some $q \in$ $\left[\frac{2 d}{d-1}, \frac{2(d+1)}{d-1}\right]$.

Definition 3.1. Given $p>2$ and $\gamma>0$, we say that hypothesis $\mathcal{H}^{\text {str }}(p, \gamma)$ holds if there exists $C_{\gamma}>0$ so that for any $\delta \leq \delta_{0}$ and any $f=\sum_{k} f_{k}$ with supp $\widehat{f}_{k} \subset \Pi_{k}^{(\delta)}$

$$
\|f\|_{p} \leq C_{\gamma} N^{\beta(p)+\gamma}\left(\sum_{k}\left\|f_{k}\right\|_{p}^{2}\right)^{1 / 2} .
$$

It is our objective to prove this 'strong' inequality $\mathcal{H}(p, \gamma)$ for all $\gamma>0$, in the asserted range $p \geq q+4 /(d-1)$. We formulate a weaker condition which can be seen as an analogue of a restricted weak type inequality.

Definition 3.2. Given $p>2$ and $\gamma>0$, we say that hypothesis $\mathcal{H}(p, \gamma)$ holds if there exists $C_{\gamma}>0$ so that for all $\delta=N^{-1} \leq \delta_{0}$, for all pairs of $N$-cubes $Q_{0}, Q_{0}^{\prime}$, for all $E \subset \mathcal{Z}\left(\delta^{1 / 2}\right)$, for all stable $\left(N, Q_{0}, E\right)$-packets $f$ with plate family $\mathcal{P}(f)$, and for all $\lambda \in\left(N^{\frac{d-1}{4}-\frac{1}{2(p-q)}}, N^{\frac{d-1}{4}}\right)$

$$
\left|\left\{x \in Q_{0}^{\prime}:|f(x)|>\lambda\right\}\right| \leq C_{\gamma} \lambda^{-p} N^{(\beta(p)+\gamma) p} \frac{N^{(d+1) / 2} \# \mathcal{P}(f)}{\# E} .
$$

Proposition 3.3. Let $0<\gamma<\gamma_{1}$. Then

$$
\mathcal{H}^{\text {str }}(p, \gamma) \Longrightarrow \mathcal{H}(p, \gamma) \Longrightarrow \mathcal{H}^{\text {str }}\left(p, \gamma_{1}\right)
$$

The main task in Wolff's bootstrapping procedure will then be to prove the following

Theorem 3.4. Let $d \geq 2, p>p_{d}=q+4 /(d-1)$ and $\gamma_{0}>0$. Let $\varepsilon_{0}$ be as in (2.1). If hypothesis $\mathcal{H}^{\text {str }}\left(p, \gamma_{0}\right)$ holds, then hypothesis $\mathcal{H}(p, \gamma)$ holds for all $\gamma>\left(1-\frac{\epsilon_{0}}{4}\right) \gamma_{0}$.

Indeed, if Theorem 3.4 holds, then Proposition 3.3 together with an iteration gives the validity of the strong type estimate $\mathcal{H}^{\text {str }}(p, \epsilon)$ for all $\epsilon>0$. The proof of Theorem 3.4 is given in $\S 6$, after preparation in $\S 4$ and $\S 5$.

Proof of Proposition 3.3. Note that implication $\mathcal{H}^{\text {str }}(p, \gamma) \Longrightarrow \mathcal{H}(p, \gamma)$ is immediate by Čebyšev's inequality and the convexity bound (2.7) (together with Lemma 2.4). We now show the proof of the main implication $\mathcal{H}(p, \gamma) \Longrightarrow \mathcal{H}^{\text {str }}\left(p, \gamma_{1}\right)$ for $\gamma_{1}>\gamma$.

We first establish that the restriction on $\lambda$ is superfluous. First, for an $\left(N, Q_{0}, E\right)$ packet $f$ we have $\|f\|_{\infty} \lesssim N^{(d-1) / 4}$ and by decomposing into a bounded number of subpackets we may assume that $\|f\|_{\infty}<N^{(d-1) / 4}$. In this case the set $\{x:|f(x)|>\lambda\}$ has measure zero if $\lambda \geq N^{(d-1) / 4}$.

Next, by Čebyšev's inequality and hypothesis $S(2, q)$

$$
\text { meas }(\{x:|f(x)|>\lambda\}) \leq \lambda^{-q}\|f\|_{q}^{q} \lesssim C_{\varepsilon} \lambda^{-q} N^{\left(\frac{\alpha(q)}{2}+\varepsilon\right) q}\|f\|_{q, 2 ; \delta}^{q},
$$


and by Lemma 2.4, we have $\|f\|_{q, 2 ; \delta}^{q} \leq N^{(d+1) / 2} \# \mathcal{P}(f) / \# \mathcal{E}$ since $f$ is an $\left(N, Q_{0}, E\right)$-packet.

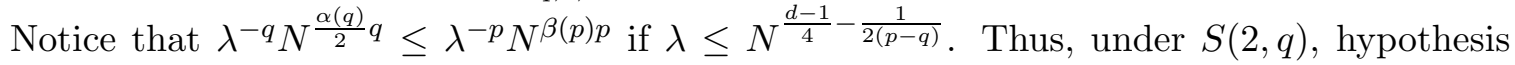
$\mathcal{H}(p, \gamma)$ implies the inequality (3.2) for all $\lambda>0$, provided $\gamma$ is replaced by $\gamma+\varepsilon$ for any $\varepsilon>0$.

We now argue that assuming $\mathcal{H}(p, \gamma)$ it suffices to show

$$
\left(\int_{Q^{\prime}}|f(x)|^{p} d x\right)^{1 / p} \leq C_{\epsilon} N^{\beta(p)+\gamma+\epsilon}\|f\|_{p, 2 ; \delta}
$$

for all $\epsilon>0$. Indeed once (3.4) is shown uniformly for all cubes we choose a grid $\mathcal{Q}$ of $N$-cubes and decompose $f=\sum \psi_{Q}^{2} f$. Notice that $\left\|\psi_{Q} f\right\|_{p, 2 ; 2 \delta} \lesssim\|f\|_{p, 2 ; \delta}$. If $Q, Q^{\prime} \in \mathcal{Q}$ for any $M_{1}>0$ then we use the estimate

$$
\left\|f \psi_{Q}^{2}\right\|_{L^{p}\left(Q^{\prime}\right)} \leq C\left(M_{1}\right)\left(\left(1+\operatorname{dist}\left(Q, Q^{\prime}\right)\right)^{-M_{1}}\left\|f \psi_{Q^{\prime}}\right\|_{p} .\right.
$$

From this it is straightforward to deduce (with $N^{\varepsilon} Q$ denoting the cube dilated by $N^{\varepsilon}$ with respect to its center) that

$$
\begin{gathered}
\left(\sum_{Q^{\prime}}\left\|\sum_{Q \in \mathcal{Q}} f \psi_{Q}\right\|_{L^{p}\left(Q^{\prime}\right)}^{p}\right)^{1 / p} \lesssim\left(\sum_{Q}\left\|f \psi_{Q}\right\|_{L^{p}\left(N^{\epsilon / 2 d} Q\right)}^{p}\right)^{1 / p}+C\left(M_{2}, \epsilon\right) N^{-M_{2}}\|f\|_{p} \\
\leq\left(\sum_{Q} \sum_{\substack{Q^{\prime} \\
\operatorname{dist}\left(Q, Q^{\prime}\right) \leq N^{1+\epsilon / 3 d}}}\left\|f \psi_{Q}\right\|_{L^{p}\left(Q^{\prime}\right)}^{p}\right)^{1 / p}+C\left(M_{2}, \epsilon\right) N^{-\frac{M_{2} \epsilon}{2 d}+d}\|f\|_{p, 2 ; \delta} .
\end{gathered}
$$

We apply (3.4) to $\psi_{Q} f$ and cubes $Q^{\prime}$ with distance $\leq N^{1+\epsilon / 2 d}$ to $Q$ and estimate the first term on the right hand side by a constant times

$$
N^{\beta(p)+\gamma+\epsilon}\left(\sum_{Q}\left\|f \psi_{Q}\right\|_{p, 2 ; 2 \delta}^{p}\right)^{1 / p} \lesssim N^{\beta(p)+\gamma+\epsilon}\|f\|_{p, 2 ; \delta} .
$$

For the last estimate we have used Lemma 2.2.

We now proceed to show (3.4). To do this we may assume

$$
\|f\|_{p, 2 ; \delta}=1 \text {. }
$$

Fix an $N$-cube $Q$. Then

$$
\|f\|_{L^{p}(Q)}^{p} \lesssim p \sum_{\ell} 2^{\ell p} \text { meas }\left(\left\{x \in Q:|f|>2^{\ell}\right\}\right)
$$

By (3.6) and (2.6) for $p=\infty$ we have that $\|f\|_{\infty} \lesssim N^{(d-1) / 4}$ so that the set where $|f|>2^{\ell}$ is empty when $2^{\ell} \gg N^{(d-1) / 4}$. Moreover, as the measure of $Q$ is $O\left(N^{d}\right)$ we have

$$
\sum_{2^{\ell} \leq N^{-d}} 2^{\ell p} \text { meas }\left(\left\{x \in Q:|f|>2^{\ell}\right\}\right) \lesssim N^{-d(p-1)}
$$

thus only the $O(\log N)$ terms with $N^{-d} \lesssim 2^{\ell} \lesssim N^{d}$ have to be estimated. 
This means that it suffices to show, for $N^{-d} \leq \lambda \leq N^{d}$,

$$
\operatorname{meas}(\{x \in Q:|f|>\lambda\}) \lesssim \lambda^{-p} N^{(\beta(p)+\gamma+\varepsilon) p}
$$

$c f$. the normalization (3.6). This normalization (together with (2.5)) also implies $\|f\|_{\infty, 2 ; \delta} \lesssim$ $N^{-(d+1) / 2 p}$. We now use the decomposition in Lemma 2.5 with $A \approx N^{-(d+1) / 2 p}$. The function $g$ in $(2.16)$ is then $\lesssim N^{-9 d} \ll \lambda$. By the pidgeonhole principle applied to the $O\left((\log N)^{3}\right)$ terms in the sum in $(2.16)$ there is a set $E_{*} \subset \mathcal{Z}\left(N^{1 / 2}\right)$, a stable $\left(N, Q, E_{*}\right)$ packet $f_{*}$, a number $j_{*}$ with $N^{-11 d} \lesssim 2^{j_{*}} \lesssim 1$ and a constant $C$ so that $2^{j_{*} p} N^{\frac{d+1}{2}} \# \mathcal{P}\left(f_{*}\right) \lesssim$ $\# E_{*}$, and

$$
\text { meas }\{x \in Q:|f|>\lambda\} \lesssim(\log N)^{3} \text { meas }\left(\left\{x \in Q: 2^{j^{*}}\left|f_{*}\right|>\lambda(\log N)^{-3} C^{-1}\right\}\right) .
$$

By Hypothesis $\mathcal{H}(p, \gamma)$ (and our initial observation that the restriction on $\lambda$ in this hypothesis is superfluous) the right hand side is estimated by a constant times

$$
(\log N)^{3} N^{(\beta(p)+\gamma) p}\left(\lambda 2^{-j_{*}}(\log N)^{-3}\right)^{-p} \frac{N^{(d+1) / 2} \# \mathcal{P}\left(f_{*}\right)}{\# E_{*}} \leq C_{\varepsilon} \lambda^{-p} N^{(\beta(p)+\gamma+\varepsilon) p},
$$

where in the last step we have used the key inequality (2.18) and $\|f\|_{p, 2 ; \delta}=1$. This finishes the proof of (3.7) and thus the proposition.

\section{LOCALiZATion}

This section is included for expository reasons; it is essentially taken from [7], with minor modifications. The purpose is to identify, for given $\lambda$, properties of specific plate families so that the improvement in Theorem 3.4 holds.

We begin with an easy localization estimate which will later give a crucial gain in the induction on scales argument.

Lemma 4.1. Let $\widehat{f}$ be supported in $\Sigma^{\delta}$ and let $Q$ be a cube of diameter $\rho \delta^{-1}$ (here $\left.\rho \leq 1\right)$. Then

$$
\left\|\psi_{Q} f\right\|_{2} \lesssim \rho^{1 / 2}\|f\|_{2}
$$

Proof. By Plancherel's theorem this is equivalent with a statement about the integral operator $T$ with kernel $K_{\delta}(\xi, \eta)=\widehat{\psi_{Q}}(\xi-\eta) \chi_{\Sigma^{\delta}}(\eta)$. Let $A_{1}=\sup _{\xi} \int\left|K_{\delta}(\xi, \eta)\right| d \eta$ and $A_{2}=\sup _{\eta} \int\left|K_{\delta}(\xi, \eta)\right| d \xi$. Then the $L^{2}$ operator norm of $T$ is $\leq \sqrt{A_{1} A_{2}}$. Now clearly $A_{2}=O(1)$ while the smaller $\eta$-support yields $A_{1}=O(\rho)$. This implies the assertion.

We now state a definition of localization for packets.

Definition 4.2. Let $R$ be an $N$-cube and let $f$ be an $(N, R, E)$-packet and $t=\delta^{\varepsilon_{0}}$ with $0<\varepsilon_{0} \ll 1 / 2$. We say that $f$ localizes at height $\lambda$ (with respect to $t N$ cubes) if there are 
subpackets $f^{Q}$ of $f$ where $Q$ runs over $t N$-cubes in a grid $\mathcal{Q}$, such that

$$
\sum_{Q} \# \mathcal{P}\left(f^{Q}\right) \lesssim \# \mathcal{P}(f)
$$

and

$$
\text { meas }(\{x:|f(x)|>\lambda\}) \lesssim \sum_{Q} \text { meas }\left(Q \cap\left\{x:\left|f^{Q}\right| \gtrsim \lambda\right\}\right) \text {. }
$$

Lemma 4.3. Let $p>2$ and suppose that $\mathcal{H}^{\text {str }}\left(p, \gamma_{0}\right)$ holds. Let $f$ be a stable $(N, R, E)$ packet and assume that $f$ localizes at height $\lambda$ (with respect to $t N=\delta^{\varepsilon_{0}-1}$ cubes), and let the $f^{Q}$ be as in Definition 4.2. Then for any $N$-cube $Q_{0}$ the estimate (3.2), i.e.

$$
\left|\left\{x \in Q_{0}:|f(x)|>\lambda\right\}\right| \leq C_{\gamma} \lambda^{-p} N^{(\beta(p)+\gamma) p} \frac{N^{(d+1) / 2} \# \mathcal{P}(f)}{\# E}
$$

holds for this $f, R$ and $\lambda$, and for all $\gamma>\gamma_{0}\left(1-\varepsilon_{0} / 2\right)$.

Proof. For each $t N$ cube $Q$, the function $t^{\frac{d-1}{4}} f^{Q} \psi_{Q}$ has Fourier transform supported in $\Sigma^{\delta / t}$, and

$$
\left\|t^{\frac{d-1}{4}} f^{Q} \psi_{Q}\right\|_{\infty, 2 ; C \delta / t} \lesssim 1
$$

Thus, we may apply $\mathcal{H}^{s t r}\left(p, \gamma_{0}\right)$, with $\delta$ replaced by $\delta / t$, and the convexity inequality (2.7) to obtain

$$
\begin{aligned}
\operatorname{meas}(\{x:|f(x)|>\lambda\}) & \lesssim \sum_{Q} \operatorname{meas}\left(\left\{\left|t^{\frac{d-1}{4}} f^{Q} \psi_{Q}\right| \gtrsim t^{\frac{d-1}{4}} \lambda\right\}\right) \\
& \lesssim \sum_{Q}\left(t^{\frac{d-1}{4}} \lambda\right)^{-p}(t N)^{\left(\beta(p)+\gamma_{0}\right) p}\left\|t^{\frac{d-1}{4}} f^{Q} \psi_{Q}\right\|_{2}^{2} \\
& =\sum_{Q} \lambda^{-p} N^{\left(\beta(p)+\gamma_{0}\right) p} t^{\gamma_{0} p} t^{-1}\left\|f^{Q} \psi_{Q}\right\|_{2}^{2}
\end{aligned}
$$

By Lemma 4.1 we have $t^{-1}\left\|f^{Q} \psi_{Q}\right\|_{2}^{2} \lesssim\left\|f^{Q}\right\|_{2}^{2} \lesssim N^{\frac{d+1}{2}} \frac{\# \mathcal{P}\left(f^{Q}\right)}{\# E}$, and therefore, summing in $Q$ and using (4.2) we see that (4.4) $\lesssim \lambda^{-p} N^{\left(\beta(p)+\gamma_{0}\left(1-\varepsilon_{0}\right)\right) p} N^{\frac{d+1}{2}} \# \mathcal{P}(f) / \# E$, which yields the assertion.

It is now important to identify situations in which the localization conditions of Definition 4.2 apply and thus the improvement of Lemma 4.3 holds. Such a situation is described in the following proposition.

Proposition 4.4. Let $p \geq 2$ and assume $\mathcal{H}\left(p, \gamma_{0}\right)$. Let $f$ be a stable $(N, R, E)$-packet so that for some $\lambda>0$

$$
\# \mathcal{P}(f) \leq t^{10 d} \lambda^{2} \# E
$$


Then $f$ localizes at height $\lambda$ to $t N$-cubes and hence (3.2) for any $N$-cube $Q_{0}$, i.e.

$$
\left|\left\{x \in Q_{0}:|f(x)|>\lambda\right\}\right| \leq C \lambda^{-p} N^{(\beta(p)+\gamma) p} \frac{N^{\frac{d+1}{2}} \# \mathcal{P}(f)}{\# E}
$$

holds for such $f$ and $\lambda$, and all $\gamma>\gamma_{0}\left(1-\varepsilon_{0} / 2\right)$.

It will be clear from the proof that the exponent $10 d$ of $t$ in (4.5) may be substantially lowered; this however seems to be of no consequence to the range of $p$ in Theorem 1.1.

The main geometrical argument behind Proposition 4.4 is in the following result from [7] which (in a slightly more complicated version) will be applied to $W=\{x:|f(x)|>\lambda\}$.

Lemma 4.5. Let $\mathcal{P}$ be a family of $N$-plates intersecting a fixed cube of diameter $C N$ and let $W$ be a measurable subset of $\mathbb{R}^{d}$. Let $t=\delta^{\varepsilon_{0}}$ and let $\mathcal{Q}$ be a grid of $t N$-cubes; we write $Q=Q(x)$ if $x \in Q$ (this is well defined apart from a set of measure 0 ). For each $\pi \in \mathcal{P}$ choose a $t N$-cube $Q_{\pi} \in \mathcal{Q}$ for which the quantity $|W \cap \pi \cap Q|$ is maximal. For a plate $\pi$ and a cube $Q \in \mathcal{Q}$ we say that $\pi \sim Q$ if $Q$ intersects the 9 -fold dilate of $Q_{\pi}$. Then

$$
\#\{Q: \pi \sim Q\} \leq 10^{d} \text { for every } \pi \in \mathcal{P}
$$

and for $\mathcal{I}=\int_{W} \sum_{\pi \in \mathcal{P}, \pi \nsim Q(x)} \chi_{\pi}(x) d x$ there is the estimate

$$
\mathcal{I} \lesssim t^{-3 d}|W| \sqrt{\# \mathcal{P}}
$$

Proof. The condition that all plates in $\mathcal{P}$ intersect a fixed $N$ cube, and the separation property of the plates implies $\# \mathcal{P}=O\left(N^{d}\right)$.

Note that (4.6) is trivial from the definition of the relation. To prove (4.7) we first note that $\mathcal{I}=\sum_{\pi} \nu(\pi)$ where $\nu(\pi)=|\{x \in W \cap \pi: Q(x) \nsim \pi\}|$. We only need to bound

$$
\widetilde{\mathcal{I}}=\sum_{\substack{\pi \in \mathcal{P}: \\ N^{-d}|W| \leq \nu(\pi) \leq|W|}} \nu(\pi)
$$

since the analogous sum involving plates $\pi \in \mathcal{P}$ with $\nu(\pi) \leq|W| N^{-d}$ is trivially bounded by $\# \mathcal{P}|W| N^{-d} \lesssim|W|$.

In (4.8) there are $O(\log N)$ relevant dyadic scales between $N^{-d}|W|$ and $|W|$ and thus we can use a pidgeonhole argument to get a subfamily $\mathcal{P}^{\prime} \subset \mathcal{P}$ and a value of $\nu$ between $N^{-d}|W|$ and $|W|$ so that

$$
|\widetilde{\mathcal{I}}| \lesssim \nu \operatorname{card}\left(\mathcal{P}^{\prime}\right) \quad \text { and } \quad \nu \leq \nu(\pi) \leq 2 \nu \quad \text { for each } \pi \in \mathcal{P}^{\prime}
$$

Hence for each $\pi \in \mathcal{P}^{\prime}$ there is a cube $Q^{\prime}(\pi)$ not related to $\pi$ so that

$$
\left|W \cap Q^{\prime}(\pi) \cap \pi\right| \gtrsim t \nu .
$$

By the maximality condition in the definition of $Q_{\pi}$ we must then also have

$$
\left|W \cap Q_{\pi} \cap \pi\right| \gtrsim t \nu \text { for each } \pi \in \mathcal{P}^{\prime} .
$$


Clearly the number of all possible pairs of $t N$ cubes is $O\left(t^{-2 d}\right)$. This means that we can find two $t N$ cubes $Q, Q^{\prime}$ in $\mathcal{Q}$ and a subfamily $\mathcal{P}^{\prime \prime}$ of $\mathcal{P}^{\prime}$ which has cardinality $\gtrsim t^{2 d} \# \mathcal{P}^{\prime}$ so that for all $\pi \in \mathcal{P}^{\prime \prime}$ we have $Q_{\pi}=Q$ and $Q^{\prime}(\pi)=Q^{\prime}$.

We now fix these two $t N$ cubes $Q$ and $Q^{\prime}$ and consider the auxiliary expression

$$
\mathcal{A}=\sum_{\pi \in \mathcal{P}^{\prime \prime}}|W \cap Q \cap \pi|\left|W \cap Q^{\prime} \cap \pi\right| .
$$

Then we have the lower bound

$$
\mathcal{A} \gtrsim(t \nu)^{2} \operatorname{card}\left(\mathcal{P}^{\prime \prime}\right) \gtrsim t^{2 d+2} \operatorname{card}\left(\mathcal{P}^{\prime}\right) \nu^{2}
$$

We can also derive an upper bound by rewriting

$$
\mathcal{A}=\int_{W \cap Q} \int_{W \cap Q^{\prime}} \sum_{\pi \in \mathcal{P}^{\prime \prime}} \chi_{\pi}(x) \chi_{\pi}\left(x^{\prime}\right) d x d x^{\prime}
$$

If $\pi \cap Q \neq \emptyset$ and $\pi \cap Q^{\prime} \neq \emptyset$ for some $\pi \in \mathcal{P}^{\prime \prime}$ then $\pi$ is related to $Q$ but not to $Q^{\prime}$, thus the distance of $Q$ to $Q^{\prime}$ is at least $t N$. This means that for each pair of points $\left(x, x^{\prime}\right) \in Q \times Q^{\prime}$ there are no more than $C t^{-d+1}$ separated plates which go through both $x$ and $x^{\prime}$. Therefore the integrand $\sum_{\pi \in \mathcal{P}^{\prime \prime}} \chi_{\pi}(x) \chi_{\pi}(x)$ is $O\left(t^{-d+1}\right)$, and hence we get the upper bound

$$
\mathcal{A} \lesssim t^{-d+1}\left|W \cap Q \| W \cap Q^{\prime}\right| \lesssim t^{-d+1}|W|^{2}
$$

Comparing the upper and the lower bounds for $\mathcal{A}$ we find that

$$
\nu \leq t^{-d-1}\left(\# \mathcal{P}^{\prime}\right)^{-1 / 2} \sqrt{\mathcal{A}} \leq t^{-(3 d+1) / 2}|W|\left(\# \mathcal{P}^{\prime}\right)^{-1 / 2}
$$

and thus using (4.9) we obtain

$$
\widetilde{\mathcal{I}} \lesssim t^{-(3 d+1) / 2}|W| \sqrt{\# \mathcal{P}^{\prime}}
$$

Unfortunately, for technical reasons Lemma 4.5 is not quite enough since we need to replace the characteristic functions $\chi_{\pi}$ by the similar weights $w_{\pi}$ with "Schwartz-tails"). This is fairly straightforward and requires adjustments in the definition of the relation between plates and $t N$-cubes and some additional pidgeonholing. We state the required estimate and refer to Lemma 4.4 in the paper by Łaba and Wolff [7] for the details of the proof.

Lemma 4.6. Let $\mathcal{P}$ be a family of $N$-plates intersecting a fixed cube of diameter $C N$ and let $W$ be a measurable subset of $\mathbb{R}^{d}$. Let $M_{0}$ be a large constant and assume that the constant $M$ in the definition of $w(x)$ is large (see (2.3)), so that $M \geq 10 M_{0} d$. Let $t=\delta^{\varepsilon_{0}}$ and let $\mathcal{Q}$ be a grid of $t N$-cubes, where again we write $Q=Q(x)$ if $x \in Q$. There is a relation $\sim$ between plates in $\mathcal{P}$ and $t N$-cubes in $\mathcal{Q}$ so that

$$
\#\{Q: \pi \sim Q\} \lesssim 1 \text { for every } \pi \in \mathcal{P}
$$


and if

$$
\mathfrak{W}_{\mathcal{P}}(x)=\sum_{\substack{\pi \in \mathcal{P} \\ \pi \nsim Q(x)}} w_{\pi}(x)
$$

then

$$
\int_{W} \mathfrak{W}_{\mathcal{P}}(x) d x \lesssim t^{-3 d}|W| \sqrt{\# \mathcal{P}}+\delta^{M_{0}}|W|
$$

Proof of Proposition 4.4. We wish to apply Lemma 4.3 and therefore have to show that with $\mathcal{P} \equiv \mathcal{P}(f)$ under the assumption $\# \mathcal{P} \leq c t^{10 d} \lambda^{2} \# E$ the localization condition in Definition 4.2 holds.

We proceed applying Lemma 4.6 to $W=\{x:|f| \geq \lambda\}$, and let $\sim$ be the relation between $N$-plates and $t N$-cubes from Lemma 4.6. Recall that $f(x)=(\# E)^{-1 / 2} \sum_{\pi \in \mathcal{P}} f_{\pi}$ with $\left|f_{\pi}\right| \lesssim w_{\pi}$. For every $t N$-cube $Q \in \mathcal{Q}$ define $f^{Q}(x)=(\# E)^{-1 / 2} \sum_{\pi \sim Q} f_{\pi}$.

By condition (4.10) we have $\sum_{Q} \# \mathcal{P}\left(f^{Q}\right) \lesssim \# \mathcal{P}(f)$, i.e. (4.2). Moreover with $\mathcal{P} \equiv \mathcal{P}(f)$

$$
\int_{W} \mathfrak{W}_{\mathcal{P}}(x) d x \lesssim t^{-3 d}|W| \sqrt{\# \mathcal{P}} \lesssim t^{-3 d}|W| \sqrt{t^{10 d} \lambda^{2} \# E} \lesssim t^{2 d}|W| \lambda \sqrt{\# E} .
$$

This means that there is a subset $W^{*}$ of $W$ so that $\left|W^{*}\right| \geq|W| / 2$ so that the pointwise bound $\mathfrak{W}_{\mathcal{P}}(x) \lesssim t \lambda \sqrt{\# E}$ for $x \in W^{*}$. Also if $x \in W^{*} \cap Q$ we have

$$
\left|f(x)-f^{Q}(x)\right|=\left|\frac{1}{\sqrt{\# E}} \sum_{\pi: \pi \nsim Q} f_{\pi}(x)\right| \lesssim \frac{\mathfrak{W}_{\mathcal{P}}(x)}{\sqrt{\# E}} \lesssim t \lambda
$$

and hence $\left|f^{Q}(x)\right| \geq \lambda$ for $x \in W^{*} \cap Q$. This implies the localization condition (4.3).

\section{A PARABOLIC RESCALING}

We first note that the paraboloid in Wolff's theorem can be replaced by $\left\{\xi: \xi_{d}=\right.$ $\left.c+\left(\xi^{\prime}-a^{\prime}\right)^{t} A\left(\xi^{\prime}-a^{\prime}\right)\right\}$ for any positive definite matrix $A$, by a linear transformation. We also may rotate the paraboloid in $\mathbb{R}^{d}$ and obtain a similar result.

More useful is the following Lemma which is an analogue and consequence of Wolff's inequality for Fourier plates in an angular sector of angle $\sqrt{\sigma} \gg \sqrt{\delta}$ (or equivalently, for $\delta$-Fourier plates contained in a fixed $\sigma$-Fourier plate).

Lemma 5.1. Let $\delta<\sigma<1$ and consider a $\sigma$-plate $\Pi^{(\sigma)}$ contained in $\Sigma^{\sigma}$. Suppose that Hypothesis $\mathcal{H}^{\text {str }}(p, \gamma)$ holds. Then for all functions $h_{k} \in L^{p}\left(\mathbb{R}^{d}\right)$

$$
\left\|\sum_{k: \Pi_{k}^{(\delta)} \subset \Pi^{(\sigma)}} P_{k}^{(\delta)} h_{k}\right\|_{p} \lesssim(\sigma / \delta)^{\beta(p)+\gamma}\left(\sum_{k}\left\|h_{k}\right\|_{p}^{2}\right)^{1 / 2} .
$$


Proof. By a rotation and translation we may assume that we are working with the standard paraboloid and the $\sigma$-plate $\Pi^{(\sigma)}=\left\{\xi:\left|\xi_{i}\right| \leq \sqrt{\sigma}, i=1, \ldots, d-1 ;\left|\xi_{d}\right| \leq \sigma\right\}$. Let $f_{k}=P_{k}^{(\delta)} h_{k}, L_{\sigma}(\xi)=\left(\sigma^{1 / 2} \xi^{\prime}, \sigma \xi_{d}\right)$ and let $f_{k}^{\sigma}(x):=\sigma^{-(d+1) / 2} f_{k}\left(L_{\sigma}^{-1} x\right)$ so that $\widehat{f_{k}^{\sigma}}(\xi)=$ $\widehat{f}_{k}\left(\sigma^{1 / 2} \xi^{\prime}, \sigma \xi_{d}\right)$. The functions $\widehat{f_{k}^{\sigma}}$ are supported in $(\delta / \sigma)^{1 / 2} \times \cdots \times(\delta / \sigma)^{1 / 2} \times \delta / \sigma$ plates tangential to the paraboloid and Hypothesis $\mathcal{H}^{\text {str }}(p, \gamma)$ yields

$$
\left\|\sum_{|k| \lesssim \sqrt{\sigma / \delta}} f_{k}^{\sigma}\right\|_{p} \lesssim(\delta / \sigma)^{-\beta(p)-\gamma}\left(\sum_{k}\left\|f_{k}^{\sigma}\right\|_{p}^{2}\right)^{1 / 2} .
$$

Changing variables $y=L_{\sigma}^{-1} x$ on both sides yields the assertion.

\section{Proof of Theorem 3.4}

Let $R$ be an $N$-cube, let $p>q+4 /(d-1)$ and $\varepsilon_{0}$ be as in (2.1). We also fix $0<\varepsilon_{1} \leq 10^{-2} \varepsilon_{0}$. Assuming that $\mathcal{H}^{\text {str }}\left(p, \gamma_{0}\right)$ holds we need to show for any stable $(N, R, E)$-packet $f$ and any fixed $N$-cube $Q_{0}$ that

$$
\text { meas }\left(\left\{x \in Q_{0}:|f(x)|>\lambda\right\}\right) \leq C_{\gamma} \lambda^{-p} N^{(\beta(p)+\gamma) p} N^{(d+1) / 2} \frac{\# \mathcal{P}(f)}{\# E}
$$

for all $\gamma>\gamma_{0}\left(1-\varepsilon_{0} / 4\right)$ and all $\lambda$ in the range

$$
N^{\frac{d-1}{4}-\frac{1}{2(p-q)}} \lesssim \lambda \lesssim N^{\frac{d-1}{4}}
$$

This will be done by localizing at a smaller scale $N_{1}$ and then using the induction hypothesis at that scale. We may without loss of generality assume that $\operatorname{dist}\left(R, Q_{0}\right) \leq 2 N^{1+\varepsilon_{1}}$ (otherwise a much better inequality holds).

Let $N_{1}$ be a number with

$$
\sqrt{N} \leq N_{1} \ll N
$$

we shall later see that the choice $N_{1}=\sqrt{N}$ will be optimal for our proof. Set $\delta_{1}=N_{1}^{-1}$ and let $\{\Delta\}$ be a tiling of $\mathbb{R}^{d}$ by $N_{1}$-cubes. For each such $\Delta$ let $\widetilde{\Delta}$ be a cube with same center as $\Delta$ but with sidelength equal to $5 N_{1}^{1+\varepsilon_{1}}$.

Now since $\min _{x \in Q} \psi_{Q}(x) \geq c>0$ with a universal constant $c$ we have

$$
\left|\left\{x \in Q_{0}:|f(x)|>\lambda\right\}\right| \leq \sum_{\Delta: \Delta \cap Q_{0} \neq \emptyset}\left|\left\{x \in \Delta:\left|f \psi_{\Delta}(x)\right|>c \lambda\right\}\right|
$$

for some constant $c>0$. Given a fixed $\Delta$, the function $f \psi_{\Delta}$ has Fourier transform supported in $\Sigma^{c \delta_{1}}$. Note that $f \psi_{\Delta}$ is in general not a packet. However, by Lemma $2.5, f \psi_{\Delta}$ can be decomposed on $\Delta$ in terms of $N_{1}$-packets: 
Lemma 6.1. Let $R$ and $Q_{0}$ be $N$-cubes as above, let $f$ be an $(N, R, E)$-packet and let $\lambda$ be as in (6.2). Then there exists $\lambda_{1}>0$ so that for every $N_{1}$-cube $\Delta$ which intersects $Q_{0}$ there is a plate family $\mathcal{P}_{\Delta}$, a set $E_{\Delta} \subset \mathcal{Z}\left(N_{1}^{1 / 2}\right)$, and a stable $\left(N_{1}, \widetilde{\Delta}, E_{\Delta}\right)$-packet $f_{\Delta}$ so that

$$
\left|\left\{x \in Q_{0}:|f(x)|>\lambda\right\}\right| \lesssim \sum_{\Delta \cap Q_{0} \neq \emptyset}\left|\left\{x \in \Delta:\left|f_{\Delta}(x)\right| \geq \lambda_{1}\right\}\right|
$$

and

$$
\frac{\# \mathcal{P}_{\Delta}}{\# E_{\Delta}} \lesssim \frac{\lambda_{1}^{2}}{\lambda^{2}} \frac{\left\|f \psi_{\Delta}\right\|_{2}^{2}}{N_{1}^{\frac{d+1}{2}}} \lesssim \frac{\lambda_{1}^{2}}{\lambda^{2}} N_{1}^{\frac{d-1}{2}}
$$

Moreover, for $2 \leq p<\infty$,

$$
\frac{\# \mathcal{P}_{\Delta}}{\# E_{\Delta}} \lesssim \frac{\lambda_{1}^{p}}{\lambda^{p}} \frac{\left\|f \psi_{\Delta}\right\|_{p, 2 ; \delta_{1}}^{p}}{N_{1}^{\frac{d+1}{2}}}
$$

Proof. Fix an $N_{1}$ cube $\Delta$ intersecting $Q_{0}$ and let $g \equiv g^{\Delta}=f \psi_{\Delta}$, which has Fourier transform supported in $\Sigma^{c \delta_{1}}$ and satisfies

$$
\left\|g^{\Delta}\right\|_{\infty, 2 ; c \delta_{1}} \lesssim\left(N / N_{1}\right)^{(d-1) / 4}=A .
$$

By Lemma 2.5 we can write

$$
g^{\Delta}(x)=C \sum_{N_{1}^{-10 d} \lesssim 2^{j} \lesssim N_{1}^{d}} 2^{j} \sum_{\ell=1}^{n_{j, \Delta}} g_{[j, \ell]}^{\Delta}(x)+h^{\Delta}(x), \quad x \in \Delta,
$$

where

$$
\begin{aligned}
& \sup _{x \in \Delta}\left|h_{\Delta}(x)\right| \leq C_{\varepsilon_{1}} N_{1}^{-8 d} A, \\
& n_{j, \Delta} \leq C_{\varepsilon_{1}}\left(\log N_{1}\right)^{2}
\end{aligned}
$$

moreover, for each $(j, \ell, \Delta)$ there is a subset $E_{j, \ell}^{\Delta}$ of $\mathcal{Z}\left(N_{1}^{1 / 2}\right)$ so that $g_{(j, \ell)}^{\Delta}$ is a stable $\left(N_{1}, \widetilde{\Delta}, E_{j, \ell}^{\Delta}\right)$-packet, with associated plate family $\mathcal{P}_{j, \ell}^{\Delta}$, which contains only $N_{1}$-plates $\pi$ with $\operatorname{dist}(\Delta, \pi) \lesssim N_{1}^{1+\varepsilon_{1}}$, and

$$
2^{j p} N_{1}^{\frac{d+1}{2}} \# \mathcal{P}_{j, \ell}^{\Delta} \lesssim\left\|f \psi_{\Delta}\right\|_{p, 2 ; \delta_{1}}^{p} \# E_{j, \ell}^{\Delta}, \quad 2 \leq p<\infty .
$$

As there are only $O(\log N)$ values of $j$ and $O\left((\log N)^{2}\right)$ values of $\ell$ a simple pidgeonhole argument shows for $\lambda$ in the range (6.2)

$$
\begin{aligned}
\left|\left\{x \in \Delta:\left|g^{\Delta}\right|>c \lambda\right\}\right| & \leq\left|\left\{x \in \Delta:\left|\sum_{N_{1}^{-10 d}} \lesssim_{2^{j} \lesssim N_{1}^{d}} 2^{j} \sum_{\ell=1}^{n_{j, \Delta}} g_{[j, \ell]}^{\Delta}(x)\right|>\frac{c \lambda}{2}\right\}\right| \\
& \leq\left|\left\{x \in \Delta:\left|2^{j_{\Delta}} g_{\left[j_{\Delta}, \ell_{\Delta}\right]}^{\Delta}(x)\right|>\frac{\lambda}{C(\log N)^{3}}\right\}\right|
\end{aligned}
$$

for some fixed $j_{\Delta}, \ell_{\Delta}$. 
Pigeonholing once again we can find, among the $\left(j_{\Delta}, \ell_{\Delta}\right)$ 's, a fixed $j_{*}, \ell_{*} \in \mathbb{Z}$ (independent of $\Delta$ ) so that

$$
\sum_{\Delta}\left|\left\{x \in \Delta:\left|g^{\Delta}\right|>c \lambda\right\}\right| \leq C(\log N)^{3} \sum_{\Delta}\left|\left\{x \in \Delta:\left|2^{j_{*}} g_{\left[j_{*}, \ell_{*}\right]}^{\Delta}(x)\right|>\frac{\lambda}{C(\log N)^{3}}\right\}\right| .
$$

This means that (6.5) holds with $\lambda_{1}=2^{-j_{*}} \lambda /(C \log N)^{3}, f_{\Delta}=g_{\left[j_{*}, \ell_{*}\right]}^{\Delta}, E_{\Delta}=E_{j_{*}, \ell_{*}}^{\Delta}$ and $\mathcal{P}_{\Delta}=\mathcal{P}\left(g_{\left[j_{*}, \ell_{*}\right]}^{\Delta}\right)$.

To prove (6.7) just observe that, by (6.11)

$$
\frac{\# \mathcal{P}_{\Delta}}{\# E_{\Delta}} \lesssim 2^{-j_{*} p} N_{1}^{-(d+1) / 2}\left\|f \psi_{\Delta}\right\|_{p, 2 ; \delta_{1}}^{p} \approx(\log N)^{3 p} \frac{\lambda_{1}^{p}}{\lambda^{p} N_{1}^{\frac{d+1}{2}}}\left\|f \psi_{\Delta}\right\|_{p, 2 ; \delta_{1}}^{p} .
$$

The first inequality in (6.6) follows from the case $p=2$ of (6.7). For the second inequality in (6.6) we observe that if $f=\sum_{k} f_{k}$ with supp $\widehat{f}_{k} \subset \Pi_{k}^{(\delta)}$ then the Fourier transforms $\widehat{f_{k} \psi_{\Delta}}$ are supported in essentially disjoint $C \sqrt{\delta}$-cubes (here we use that $N_{1} \geq \sqrt{N}$ ). Thus we have the crucial orthogonality estimate

$$
\left\|f \psi_{\Delta}\right\|_{2}^{2} \lesssim \sum_{k}\left\|f_{k} \psi_{\Delta}\right\|_{2}^{2} \lesssim|\Delta| \sum_{k \in E}\left\|f_{k}\right\|_{\infty}^{2} \lesssim N_{1}^{d}
$$

since $f$ was assumed to be an $(N, R, E)$-packet. The second inequality in (6.6) follows.

We wish to use the bound in (6.6) to argue that Proposition 4.4 can be applied to the pair $\left(f_{\Delta}, \lambda_{1}\right)$. The next lemma, shows how to conclude the theorem for $(f, \lambda)$ in such case. Basically, one rescales the problem and uses one more time the induction hypothesis at scale $N / N_{1}$.

Lemma 6.2. Let $p>2$ and assume $\mathcal{H}^{\text {str }}\left(p, \gamma_{0}\right)$. Let $f$ be a $(N, R, E)$-packet for some $N$-cube $R$, let $Q_{0}$ be an $N$-cube and let $\lambda$ as in (6.2). Let $\omega>0$ and suppose that for every $N_{1}$-cube $\Delta$ intersecting $Q_{0}$, the quadruplet $\left(f_{\Delta}, \mathcal{P}_{\Delta}, E_{\Delta}, \lambda_{1}\right)$ defined in Lemma 6.1 satisfies

$$
\left|\left\{x \in \Delta:\left|f_{\Delta}(x)\right|>\lambda_{1}\right\}\right| \lesssim \frac{N_{1}^{(\beta(p)+\omega) p}}{\lambda_{1}^{p}} N_{1}^{\frac{d+1}{2}} \frac{\# \mathcal{P}_{\Delta}}{\# E_{\Delta}} .
$$

Then, we also have

$$
\left|\left\{x \in Q_{0}:|f(x)|>\lambda\right\}\right| \lesssim \lambda^{-p} \frac{N^{\left(\beta(p)+\gamma_{0}\right) p}}{N_{1}^{\left(\gamma_{0}-\omega\right) p}} N^{\frac{d+1}{2}} \frac{\# \mathcal{P}(f)}{\# E} .
$$

This is saying that if we have an improvement in (6.13) with an $\omega<\gamma_{0}$ then we also get an improvement in our main bound (6.14).

Proof of Theorem 3.4, given Lemma 6.2. We choose $N_{1}=\sqrt{N}$. We need to verify that (6.13) holds with $\omega>\gamma\left(1-\varepsilon_{0} / 2\right)$. Then Lemma 6.2 tells us that (6.14) holds with $\beta>\gamma\left(1-\varepsilon_{0} / 4\right)$ (where, say, $\varepsilon_{0}$ is chosen as in (2.1)). Proposition 4.4 says that (6.13) holds 
if the plate families $\mathcal{P}_{\Delta}$ satisfy $\# \mathcal{P}_{\Delta} \lesssim t_{1}^{10 d} \lambda_{1}^{2} \# E_{\Delta}$ where $t_{1}=\delta_{1}^{\varepsilon_{0}}$. By (6.6) and the lower bound on $\lambda, \lambda \gtrsim N^{\frac{d-1}{4}-\frac{1}{2(p-q)}}$ we have

$$
\lambda_{1}^{-2} \frac{\# \mathcal{P}_{\Delta}}{\# E_{\Delta}} \lesssim N_{1}^{(d-1) / 2} \lambda^{-2}=N^{(d-1) / 4} \lambda^{-2} \lesssim N^{\frac{1}{p-q}-\frac{d-1}{4}}
$$

and we are done if $N^{\frac{1}{p-q}-\frac{d-1}{4}} \lesssim t_{1}^{10 d}=N^{-5 d \varepsilon_{0}}$. This holds if $1 /(p-q)-(d-1) / 4<-5 d \varepsilon_{0}$ or equivalently $p>q+4 /\left(d-1-20 d \varepsilon_{0}\right)$. Note that this inequality is implied by $(2.1)$ (and that the precise choice of $\varepsilon_{0}$ is not important in the argument).

Proof of Lemma 6.2. By (6.5) and (6.13) we have

$$
\begin{aligned}
\left|\left\{x \in Q_{0}:|f|>\lambda\right\}\right| & \lesssim \sum_{\Delta}\left|\left\{x \in \Delta:\left|f_{\Delta}\right|>\lambda_{1}\right\}\right| \\
& \lesssim \sum_{\Delta} \lambda_{1}^{-p} N_{1}^{(\beta(p)+\omega) p} N_{1}^{\frac{d+1}{2}} \frac{\# \mathcal{P}_{\Delta}}{\# E_{\Delta}} .
\end{aligned}
$$

Thus, the result will be established if we can show

$$
\sum_{\Delta} N_{1}^{\frac{d+1}{2}} \frac{\# \mathcal{P}_{\Delta}}{\# E_{\Delta}} \lesssim \frac{\lambda_{1}^{p}}{\lambda^{p}}\left(N / N_{1}\right)^{\left(\beta(p)+\gamma_{0}\right) p} N^{\frac{d+1}{2}} \frac{\# \mathcal{P}(f)}{\# E}
$$

Now consider functions $\Xi_{l}$ so that their Fourier transforms $\widehat{\Xi}_{l}$ are bump functions associated to the $\delta_{1}^{1 / 2} \times \ldots \times \delta_{1}^{1 / 2} \times \delta_{1}$-plates $\Pi_{l}^{\delta_{1}}$. Then by (6.7) we have for each $\Delta$,

$$
\begin{aligned}
& N_{1}^{\frac{d+1}{2}} \frac{\# \mathcal{P}_{\Delta}}{\# E_{\Delta}} \lesssim \frac{\lambda_{1}^{p}}{\lambda^{p}}\left\|f \psi_{\Delta}\right\|_{p, 2 ; \delta_{1}}^{p} \lesssim \frac{\lambda_{1}^{p}}{\lambda^{p}}\left(\sum_{l}\left\|\left(f \psi_{\Delta}\right) * \Xi_{l}\right\|_{p}^{2}\right)^{p / 2} \\
& \lesssim \frac{\lambda_{1}^{p}}{\lambda^{p}}\left(\sum_{l}\left\|\left[\psi_{\Delta}\left(\sum_{k: \Pi_{k}^{(\delta)} \subset C \Pi_{l}^{\left(\delta_{1}\right)}} f_{k}\right)\right] * \Xi_{l}\right\|_{p}^{2}\right)^{p / 2} \lesssim \frac{\lambda_{1}^{p}}{\lambda^{p}}\left(\sum_{l}\left\|\psi_{\Delta}\left(\sum_{\Pi_{k}^{(\delta)} \subset C \Pi_{l}^{\left(\delta_{1}\right)}} f_{k}\right)\right\|_{p}^{2}\right)^{p / 2} .
\end{aligned}
$$

We sum in $\Delta$ and apply Minkowski's inequality to obtain

$$
\begin{aligned}
& \sum_{\Delta} N_{1}^{\frac{d+1}{2}} \frac{\# \mathcal{P}_{\Delta}}{\# E_{\Delta}} \lesssim \frac{\lambda_{1}^{p}}{\lambda^{p}} \sum_{\Delta}\left(\sum_{l}\left\|\psi_{\Delta}\left(\sum_{\Pi_{k}^{(\delta)} \subset C \Pi_{l}^{\left(\delta_{1}\right)}} f_{k}\right)\right\|_{p}^{2}\right)^{p / 2} \\
& \lesssim \frac{\lambda_{1}^{p}}{\lambda^{p}}\left(\sum_{l}\left[\sum_{\Delta}\left\|\psi_{\Delta}\left(\sum_{\Pi_{k}^{(\delta)} \subset C \Pi_{l}^{\left(\delta_{1}\right)}} f_{k}\right)\right\|_{p}^{p}\right]^{2 / p}\right)^{p / 2} \lesssim \frac{\lambda_{1}^{p}}{\lambda^{p}}\left(\sum_{l}\left\|\sum_{\Pi_{k}^{(\delta)} \subset C \Pi_{l}^{\left(\delta_{1}\right)}} f_{k}\right\|_{p}^{2}\right)^{p / 2} .
\end{aligned}
$$

Now, we apply Hypothesis $\mathcal{H}^{\text {str }}\left(p, \gamma_{0}\right)$ in the rescaled version of Lemma 5.1 and bound for each $l$

$$
\left\|\sum_{k: \Pi_{k}^{(\delta)} \subset C \Pi_{l}^{\left(\delta_{1}\right)}} f_{k}\right\|_{p} \lesssim\left(N / N_{1}\right)^{\beta(p)+\gamma_{0}}\left(\sum_{k: \Pi_{k}^{(\delta)} \subset C \Pi_{l}^{\left(\delta_{1}\right)}}\left\|f_{k}\right\|_{p}^{2}\right)^{1 / 2}
$$


This yields, using the convexity inequality $(2.7)$ and $\|f\|_{\infty, 2 ; \delta} \lesssim 1$,

$$
\begin{aligned}
& \left(\sum_{l}\left\|\sum_{\Pi_{k}^{(\delta)} \subset c \Pi_{l}^{(\delta)}} f_{k}\right\|_{p}^{2}\right)^{p / 2} \lesssim\left(N / N_{1}\right)^{\left(\beta(p)+\gamma_{0}\right) p}\left(\sum_{l} \sum_{\Pi_{k}^{(\delta)} \subset c \Pi_{l}^{\left(\delta_{1}\right)}}\left\|f_{k}\right\|_{p}^{2}\right)^{p / 2} \\
& \lesssim\left(N / N_{1}\right)^{\left(\beta(p)+\gamma_{0}\right) p} \sum_{k}\left\|f_{k}\right\|_{2}^{2}\left(\sum_{k^{\prime}}\left\|f_{k^{\prime}}\right\|_{\infty}^{2}\right)^{(p-2) / 2} \lesssim\left(N / N_{1}\right)^{\left(\beta(p)+\gamma_{0}\right) p} N^{\frac{d+1}{2}} \frac{\# \mathcal{P}(f)}{\# E},
\end{aligned}
$$

and thus we get the asserted (6.15).

\section{REFERENCES}

[1] D. Békollé, A. Bonami, G. Garrigós And F. Ricci. Littlewood-Paley decompositions related to symmetric cones and Bergman projections in tube domains. Proc. London Math. Soc. (3) 89 (2004), 317-360.

[2] J. Bourgain. Besicovitch type maximal operators and applications to Fourier analysis, Geometric and Functional analysis, 147-187.

[3] C. Fefferman. A note on spherical summation multipliers. Israel J. Math. 15 (1973), 44-52.

[4] G. Garrigós, W. Schlag, A. Seeger. Improvements in Wolff's inequality for decompositions of cone multipliers.

[5] G. Garrigós, A. Seeger, On plate decompositions for cone multipliers, to appear in Proc. Edinburgh Math. Soc.

[6] I. Łaba And M. Pramanik. Wolff's inequality for hypersurfaces. Proceedings of the 7th International Conference on Harmonic Analysis and Partial Differential Equations, El Escorial, 2004. Collect. Math. 2006, Vol. Extra, 293-326.

[7] I. ŁabA And T. WolfF. A local smoothing estimate in higher dimensions. J. Anal. Math. 88 (2002), 149-171.

[8] S. LEE. Improved bounds for Bochner-Riesz and maximal Bochner-Riesz operators. Duke Math. J. 122 (2004), no. 1, 205-232.

[9] M. Pramanik and A. Seeger. $L^{p}$ regularity of averages over curves and bounds for associated maximal operators. Amer. J. Math. 129 (2007), 61-103.

[10] E.M. Stein. Singular integrals and differentiability properties of functions. Princeton Mathematical Series, No. 30 Princeton University Press, Princeton, N.J. 1970.

[11] T. TAO. A sharp bilinear restrictions estimate for paraboloids. Geom. Funct. Anal. 13 (2003), no. 6, 1359-1384.

[12] T. TaO, A. Vargas and L. Vega. A bilinear approach to the restriction and Kakeya conjectures. J. Amer. Math. Soc. 11 (1998), no. 4, 967-1000.

[13] T. WolfF. Local smoothing type estimates on $L^{p}$ for large $p$. Geom. Funct. Anal. 10 (2000), 1237-1288.

G. Garrigós, Dep. Matemáticas C-XV, Universidad Autónoma de Madrid, 28049 Madrid, SPAIN

E-mail address: gustavo.garrigos@uam.es

A. Seeger, Department of Mathematics, University of Wisconsin-Madison, Madison, Wi 53706, USA

E-mail address: seeger@math.wisc.edu 